\title{
Effet de la Poudre de Piper guineense Schumach \& Thonn., 1827 (Piperaceae) sur les Paramètres Semenciers et la Mortalité de Sitophilus oryzae L., 1763 (Coleoptera Curculionidae) dans les Stocks des Semences de Riz
}

\author{
Pegalepo Esther, MA \\ Dossa Sedami Sylvie Gwladys, MA \\ Bokonon-Ganta Aimé H., PhD
}

Université d'Abomey-Calavi, Faculté des Sciences Agronomiques

(FSA/UAC), Laboratoire d'Entomologie Agricole (LEAg), Bénin

Dossou Joseph, Prof. Dr. Ir.

Université d'Abomey-Calavi, Faculté des Sciences Agronomiques

(FSA/UAC), Laboratoire de Bio-Ingénieries des Procédés Alimentaires

(LABIOPA), Bénin

Doi: 10.19044/esj.2019.v15n15p190 URL:http://dx.doi.org/10.19044/esj.2019.v15n15p190

\section{Résumé}

Le riz, est l'une des cultures vivrières hautement stratégique en Afrique et notamment au Bénin. Cependant, les contraintes du stockage obligent les producteurs à l'utilisation des pesticides chimiques qui sont très nocifs pour la santé des consommateurs. L'étude menée au laboratoire d'entomologie d'AfricaRice, vise à évaluer l'efficacité de la poudre de Piper guineense $(\mathrm{PPg})$ contre $S$. oryzae dans les stocks de semences de riz. Ainsi, des échantillons de $100 \mathrm{~g}$ des semences de 2 variétés de riz ont été traités avec 7 traitements : $0,25 \mathrm{~g} ; 0,5 \mathrm{~g} ; 0,75 \mathrm{~g} ; 1 \mathrm{~g} ; 1,4 \mathrm{~g} ; 1,85 \mathrm{~g}$ et $2,5 \mathrm{~g}$ et en 4 répétitions, puis infestés avec 20 individus adultes $S$. oryzae. L'huile de neem $(\mathrm{HN})$ $(0,5 \mathrm{ml})$ et l'échantillon non traité ont été les témoins. Les résultats montrent qu'après 3 mois de conservation, les différents traitements à la PPg ainsi que celui à l'HN ont eu un effet insecticide sur $S$. oryzae quelle que soit la variété de riz. En effet, la dose optimale qui assure 3 mois d'efficacité du biopesticide sur la variété NERICA 1 est de $1,85 \mathrm{~g}$ pour $100 \mathrm{~g}$ de riz et de $1,4 \mathrm{~g}$ pour $100 \mathrm{~g}$ de riz sur la variété WAB 56-104. L'HN a montré une durée de persistance de 3 mois sur toutes les variétés de riz. Le taux de germination des semences stockées, n'est pas influencé par la poudre de l'épice. Ce travail a permis de mettre en évidence les doses optimales et l'efficacité de la PPg selon chaque variété de riz, en vue d'une meilleure gestion de $S$. oryzae. 
Mots-clés : Taux de germination, dose optimale, Piper guineense, Sitophilus oryzae, Conservation

\title{
Effect of Piper guineense Schumach \& Thonn Powder (Piperaceae) on the Seed Parameters and on the Control of Sitophilus oryzae L. (Coleopterea: Curculionidae) in Rice Seed Stocks
}

\author{
Pegalepo Esther, MA \\ Dossa Sedami Sylvie Gwladys, MA \\ Bokonon-Ganta Aimé H., PhD
}

Université d'Abomey-Calavi, Faculté des Sciences Agronomiques (FSA/UAC), Laboratoire d'Entomologie Agricole (LEAg), Bénin

Dossou Joseph, Prof. Dr. Ir.

Université d'Abomey-Calavi, Faculté des Sciences Agronomiques (FSA/UAC), Laboratoire de Bio-Ingénieries des Procédés Alimentaires

(LABIOPA), Bénin

\begin{abstract}
Rice is one of the highly strategic food crops in Africa, particularly in Benin. However, constraints such as storage compels producers to use chemical pesticides that are very dangerous and harmful to the health and well-being of consumers. This study, conducted at AfricaRice's entomology laboratory, therefore aims to assess the persistence of Piper guineense powder for the control of $S$. oryzae in rice stock. Thus, $100 \mathrm{~g}$ samples of seeds of two rice varieties were treated with seven treatments: $0.25 \mathrm{~g} ; 0.5 \mathrm{~g} ; 0.75 \mathrm{~g} ; 1 \mathrm{~g} ; 1.4 \mathrm{~g}$; $1.85 \mathrm{~g}$ and $2.5 \mathrm{~g}$ and in 4 repetitions and then infested with 20 adults of adult $S$. oryzae and arranged in a complete random block system. Neem oil $(0.5 \mathrm{ml})$ and the untreated sample were checks. The results obtained show that after three months of storage, the different treatments with $P$. guineense powder and neem oil had an insecticidal effect on $S$. oryzae regardless of the rice variety. Indeed, the optimal dose that ensures the 3-month efficacity of the biopesticide on the NERICA 1 variety is $1.85 \mathrm{~g}$ per $100 \mathrm{~g}$ of rice and $1.4 \mathrm{~g}$ per $100 \mathrm{~g}$ of rice on the WAB 56-104 variety. The dose of neem oil showed a three-month duration of action on all rice varieties. The germination rate of the stored seeds is not influenced by the powder of the spice. This work
\end{abstract}


enabled to identify the optimal doses and persistence of $P$. guineense powder according to each rice variety, with a view to better management of S. oryzae.

Keywords: Germination rate, optimal dose, Piper guineense, Sitophilus oryzae, storage

\section{Introduction}

Le riz, Oryza sp. L. (Poaceae) est devenu l'une des productions vivrières les plus importantes au monde (MAEP, 2013), avec en 2015-2016, une superficie d'environ 158,9 millions d'hectares emblavés et une consommation de 468,907 millions de tonnes dans le monde (Statista, 2017). Au Bénin, c'est une céréale importante dont la production en paddy est passée de 124975 tonnes en 2010 à 234145 tonnes en 2014 (FAOSTAT, 2015). Au Bénin, la consommation de cette céréale est d'environ 46,1 kg / habitant $/ \mathrm{an}$ (AfricaRice, 2015). Le riz, source principale de revenus pour des millions de personnes (Gounse, 2004), présente un potentiel de rentabilité considérable (Allagbé, 2014). Malgré son importance socio-économique et alimentaire, de nombreuses contraintes dont celles liées au stockage et à la conservation postrécolte limitent le développement de la filière riz au Bénin. Au nombre des facteurs responsables des pertes au cours du stockage, les insectes occupent une place prépondérante. C'est le cas de Sitophilus oryzae L. (Coleoptera : Curculionidae), l'un des ravageurs des grains entiers stockés les plus sérieux à l'échelle mondiale (Atwal et Dhaliwal, 2002). Les pertes dues à ce charançon, peuvent atteindre 25 à $40 \%$ des récoltes (Helbig, 1995). Face aux ravages des insectes de stocks, les moyens de lutte se sont essentiellement articulés autour de l'utilisation de pesticides de synthèse. Celles-ci sont efficaces, mais présentent de nombreux inconvénients.

Face à la contrainte que constitue l'infestation des insectes, principaux ravageurs des stocks, les moyens de lutte reposent généralement sur l'utilisation d'insecticides de synthèse (Devi et Devi, 2013). Toutefois, beaucoup d'effets non désirés relatifs à leur utilisation sont rapportés (Benhalima et al., 2004). Il s'agit entre autres de la rémanence, la non biodégradabilité de certains insecticides de synthèse qui représentent de véritables problèmes pour l'environnement (Mehrpour et al., 2014), l'accoutumance des insectes, la résistance de certaines souches et les intoxications (Benhalima et al., 2004 In Gueye et al., 2011).

Pour limiter, voire éliminer ces effets néfastes liés à l'utilisation des pesticides de synthèse dans le contrôle des insectes ravageurs de stocks, la recherche d'autres alternatives s'impose. Gueye et al., (2011) rapportent que l'usage des plantes indigènes dans la conservation des récoltes a été pratiqué avant même l'apparition des insecticides de synthèse. Ainsi, selon Cheikh (2008), l'utilisation des plantes à effet répulsif et /ou insecticide constitue 
l'une des méthodes alternatives innovatrices pour protéger les stocks. Il s'agit souvent de plantes consommées dans l'alimentation humaine, comme condiment ou épice ou des plantes à vertus médicinales (Ngamo et Hance, 2007). Leurs propriétés insecticides, larvicides et ovicides, stérilisantes, antiappétentes et répulsives ont fait l'objet de nombreuses études (Djossou, 2006, Ngamo et al., 2007 ; Kouninki et al., 2007 ; Ndomo et al., 2009). Parmi ces plantes, figurent celles de la famille des Piperaceae. En effet, Abraz (2015), a scientifiquement prouvé que la poudre du poivre noir est efficace contre les insectes de stock et n'affecte pas le pouvoir germinatif des graines du niébé en stock. Aussi, Ashouri et Shayesteh (2010) ont-ils montré l'activité insecticide de la poudre du poivre noir ( $P$. nigrum) contre deux principaux ravageurs de céréale stockés Rhyzopertha dominica Fabricus, (Coleoptera : Bostrichidae) et Sitophilus granarius Linnaeus, (Coleoptera : Curculionidae). La présente étude vise donc à évaluer l'effet de la poudre de $P$. guineense Sch. \& Tonn. (Piperaceae) sur les paramètres semenciers et le contrôle de $S$. oryzae sur les semences de 2 variétés de riz pendant une durée de stockage de 3 mois.

\section{Matériel et Méthodes \\ Description du cadre de l'étude}

Les essais ont été réalisés au Centre du riz pour l'Afrique (AfricaRice) sis à la station de IITA - Bénin (Institut International d'Agriculture Tropicale) située à Togoudo $\left(6^{\circ} 28^{\prime} \mathrm{N}, 2^{\circ} 21^{\prime} \mathrm{S}, 15 \mathrm{~m}\right.$ d'altitude $)$, dans la commune d'Abomey-Calavi à $12 \mathrm{~km}$ au Nord-Ouest de Cotonou et à 2,5 km environ de la voie inter État Cotonou-Bohicon sur le tronçon de Tankpè dans le département de l'Atlantique au Sud du Bénin.

\section{Matériel}

\section{Matériel biologique}

Le matériel animal utilisé est le charançon du riz Sitophilus oryzae L., 1763 qui est un Coléoptère de la famille de Curculionidae.

Le matériel végétal utilisé comprend 2 variétés de riz Oryza sativa L., 1753, variété NERICA 1 et la variété WAB 56-104 appartenant à la famille des Poaceae et à l'ordre des Paoles. Le matériel végétal comprend également 2 plantes locales utilisées comme biopesticides à savoir : la poudre du poivre noir de Piper guineense Schumach. et Thonn., 1827 de la famille des Piperaceae ; et l'huile de neem de Azadirachta indica A. Juss., 1830 une Meliaceae utilisée comme témoin positif.

\section{Matériel technique de laboratoire}

Au cours de l'expérimentation, un broyeur électrique a été utilisé pour réduire les grains du poivre noir en poudre. Une balance à précision $0,01 \mathrm{~g}$ a été utilisée pour les différentes pesés. Les boîtes de petri de $9 \mathrm{~mm}$ de diamètres 
ont servi pour le test de germination des semences. Les pinces ont servi à disposer les grains de riz dans les boîtes de petri. Enfin, un humidimètre de marque SAKATE a permis d'enregistrer les teneurs en eau des grains avant et après stockage.

\section{Méthodes}

\section{Elevage des insectes (S. oryzae)}

Des groupes de $S$. oryzae ont été produits à partir des souches adultes prélevées sur du riz infesté dans le magasin d'AfricaRice. Ces souches ont été cultivées sur un échantillon de $10 \mathrm{~kg}$ de NERICA 8 décortiqué et non infesté contenu dans 10 pots à raison de $1 \mathrm{~kg}$ par pot. Le tout a été conservé à $28 \pm$ $1^{\circ} \mathrm{C}$ et $70 \pm 5 \%$ HR dans le laboratoire d'entomologie d'AfricaRice. Les trous d'aération de $15 \mathrm{~cm}$ de diamètre recouverts d'un tissu en popeline ont été faits sur le couvercle des pots pour empêcher la fuite des insectes. Deux (2) semaines après le début de l'infestation, les insectes adultes ont été retirés des pots. Les grains restés dans les pots pendant 30 jours ont été suivis jusqu'à l'éclosion des œufs et l'obtention d'adultes de même génération. Les charançons du riz adultes de 1-14 jours ont été recueillis dans le milieu de culture et utilisés pour infester les semences de riz au cours de l'essai.

\section{Production de la poudre du poivre noir}

Le poivre noir a été acheté chez un producteur à Malanville au Nord du Bénin où cette épice est produite en grande quantité. Après l'avoir trié, il a été réduit en poudre et utilisé immédiatement dans l'essai.

\section{Mise en place de l'essai}

\section{Traitement des semences de riz et infestation avec les insectes adultes}

Les échantillons de riz préalablement constitués ont été conservés à $4^{\circ} \mathrm{C}$ dans un congélateur pendant deux semaines pour tuer les œufs, larves, insectes et autres organismes indésirables qui seraient présents dans les échantillons, comme suggéré par Santos et al. (2014). Avant l'application des traitements, ces échantillons congelés ont été sortis et séchés à nouveau pendant quelques heures dans le but de ramener les grains à une teneur en eau idéale (9\%) pour le stockage, comme suggéré par Hayma (1989). Ainsi, la teneur en eau des grains utilisés a été maintenue en moyenne entre 9,4 \pm $0,24 \%$ et $9,8 \pm 0,07 \%$.

Les échantillons de $100 \mathrm{~g}$ de semences de riz ont été introduits dans des pots en verre $(9 \mathrm{~cm}$ de haut et de $5 \mathrm{~cm}$ de diamètre) et traités avec la poudre de $P$. guineense à des doses variables de $0,25 \mathrm{~g} ; 0,5 \mathrm{~g} ; 0,75 \mathrm{~g} ; 1 \mathrm{~g} ; 1,4$ $\mathrm{g} ; 1,85 \mathrm{~g}$ et $2,5 \mathrm{~g}$. Les pots ont été secoués manuellement jusqu'à ce que la poudre se repartisse de façon homogène sur les grains de riz. Après 30 minutes, chaque pot contenant les grains préalablement mélangés avec la 
poudre $S$. sativae a été infesté avec 20 individus d'insectes choisis au hasard. Ces pots d'élevage ont été couverts de tissu popeline pour permettre une aération adéquate en leur sein. Ce tissu est maintenu en place avec le couvercle du bocal qui est percé d'un trou de $3 \mathrm{~cm}$ de diamètre.

Deux (2) témoins ont été utilisés : un témoin négatif (T0-) qui n'a pas reçu de poudre et un témoin positif $(\mathrm{T} 0+)$ traité avec $0,5 \mathrm{ml}$ de l'huile de neem. Cette dose a été choisie par rapport aux travaux de Hazoume (2008) qui a montré l'efficacité de la dose $5 \mathrm{ml} \mathrm{d}$ 'huile de neem pour traiter $1 \mathrm{~kg}$ de riz. Cet auteur a démontré par son étude que la dose $0,5 \mathrm{ml} / 100 \mathrm{~g}$ est la dose minimale efficace ( $86 \%$ de taux de mortalité d'insectes, sans apparition de progéniture d'insectes). Quatre (04) répétitions ont été effectuées pour chaque traitement y compris les témoins et l'essai a été répété deux fois dans le temps.

Les échantillons ont été conservés dans un abri protégé, sur des étagères. Durant la conduite de l'expérimentation, l'humidité relative et la température de l'air au sein de cet abri étaient maintenus et surveillés à l'aide d'un thermo hygromètre mural de précision $0,1^{\circ} \mathrm{C}$ et $1 \%$.

\section{Dépouillement des essais}

Le dépouillement s'est déroulé au terme de 3 mois de stockage. Dans chaque pot, les insectes morts et vivants ont été dénombrés.

Effet des doses de la poudre de $\boldsymbol{P}$. guineense sur la mortalité de $S$. oryzae

Le degré d'efficacité du produit a été évalué à travers la détermination des taux de mortalité 3 mois après la mise en place de l'essai suivant chaque dose, selon la formule suivante :

\section{TM $(\%)=$ NinsM $/($ NinsM + NinsV $) \times 100$}

Avec $\mathrm{TM}=$ taux de mortalité au $3^{\mathrm{e}}$ mois ;

NinsM $=$ nombre d'insectes morts au $3^{\mathrm{e}}$ mois ;

Nins $\mathrm{V}=$ nombre d'insectes vivants au $3^{\mathrm{e}}$ mois

Effet de la poudre de $\boldsymbol{P}$. guineense sur l'émergence de $S$. oryzae

La population d'insectes émergés a été calculée au $3^{\mathrm{e}}$ mois après les traitements, suivants chaque dose, selon les formules ci-après :

$$
\text { NinsE }=(\text { NinsV }+ \text { NinsM })-\text { NinsI }
$$

NinsE $=$ Nombre d'insectes émergés au $3^{\mathrm{e}}$ mois

Nins $\mathrm{V}=$ Nombre d'insectes vivants au $3^{\mathrm{e}}$ mois

NinsM= Nombre d'insectes morts au $3^{\mathrm{e}}$ mois

NinsI $=$ Nombre d'insectes introduits au $1^{\mathrm{er}}$ jour. Dans le présent essai, ce nombre est égal à 20 . 
Détermination de la dose optimale assurant la meilleure protection des semences de riz

La dose de $P$. guineense qui a maintenu son efficacité le plus longtemps possible a été retenue comme la dose maximale qui assure la meilleure protection pour chaque variété. La dose optimale, choisie dans chaque cas, est la plus petite dose qui s'est révélée avoir un effet statistiquement identique à celui de la dose maximale pour les paramètres et le temps de stockage.

Effet de la poudre de $\boldsymbol{P}$. guineense sur la teneur en eau des semences de riz

La teneur en humidité est le critère de qualité le plus important pour le paddy et pour les semences (Juliano et Duff, 1989). Ainsi, dans le but d'apprécier l'impact des poudres du biopesticide sur la teneur en eau des semences, cette dernière a été déterminée aussi bien au niveau des échantillons traités qu'au niveau des témoins. Elle a été mesurée au début de l'expérimentation et après 3 mois de stockage, à l'aide d'un humidimètre/testeur de marque SAKATA.

\section{Effet de la poudre de $P$. guineense sur le taux de germination des semences de riz}

Pour la réalisation des tests de germination avant et après l'expérimentation, cent (100) graines de riz, ont été prélevées dans chaque bocal et réparties dans 4 boîtes de pétri de $9 \mathrm{~cm}$ de diamètre, à raison de 25 graines de riz par boîte de pétri (Dedi et Allou, 2015). Chaque boîte de pétri a été tapisé d'un papier buvard imbibé d'eau de robinet sur lequel ont été disposées les graines. Ces boîtes de pétri ont été étiquetées selon la variété, le type de traitement ( $\mathrm{T} 0+, \mathrm{T} 0$ - ou traité à l'épice) et le numéro de la répétition. Elles ont été placées dans un germoir électrique à $25^{\circ} \mathrm{C}$ et à un taux d'Humidité Relative (HR) de 96\%. Les dépouillements ont été effectués le huitième jour après ensemencement. Les semences germées ont été dénombrées. Il a été considéré comme germées celles dont les radicules étaient visibles (Dedi et Allou, 2015). Les taux de germination ont été déterminés par la formule suivante :

\section{Taux de germination $=($ Nombre de graines germées $/$ Nombre de graines totale) $\times 100$}

\section{Analyses statistiques \\ Les différentes données collectées ont été saisies dans Microsoft Excel et les analyses statistiques ont été effectuées en utilisant le logiciel $\mathrm{R}$ version}


3.4.1 (R Core Team, 2017). Pour tester l'effet des différentes doses de produits sur le taux de germination, la teneur en eau, le taux de mortalité, le nombre d'insectes émergés, une analyse de la variance sur mesures répétées à 3 facteurs (variétés, doses et temps d'observation) a été effectuée. Quant à l'effet des variétés et des doses sur les dégâts causés par les insectes, une analyse de variance à 2 facteurs (variété et dose) a été réalisée.

Par ailleurs, le test de structuration des moyennes de SNK (Student Newman - Keuls) à $5 \%$ a été effectué pour comparer les moyennes. La corrélation entre les différents paramètres étudiés a été calculée dans le but d'apprécier le degré de corrélation entre les paramètres.

\section{Résultats}

\section{Effets des doses de $P$. guineense sur la mortalité de $S$. oryzae selon les} variétés de riz

L'analyse de l'effet des différentes doses de $P$. guineense sur le taux de mortalité moyen de $S$. oryzae chez NERICA 1 a montré que 3 mois après traitement, les doses de la poudre de $P$. guineense allant de $0,5 \mathrm{~g}$ à $2,5 \mathrm{~g}$ et le traitement à l'huile de neem ont augmenté significativement le taux de mortalité de $S$. oryzae comparativement à la dose $0,25 \mathrm{~g}$ et le lot non traité (Tableau I). Le plus faible taux moyen de mortalité $(2,99 \pm 0,36 \%)$ a été enregistré dans le bocal témoin négatif. La mortalité a été complète $(100 \pm 0 \%)$ dans la dose $2,5 \mathrm{~g}$ et a été statistiquement identique aux taux de mortalités moyens obtenus pour les doses $0,75 \mathrm{~g} ; 1 \mathrm{~g} ; 1,4 \mathrm{~g}$ et $1,85 \mathrm{~g}$ de la poudre de $P$. guineense et pour le traitement à l'huile de neem.

Au niveau de WAB56-104, il ressort que l'effet des différentes doses de la poudre de $P$. guineense sur le taux de mortalité moyen de $S$. oryzae 3 mois après traitement est statistiquement significatif (Tableau I). Tous les traitements ont eu un taux de mortalité significativement supérieur à celui du témoin non traité. Toutefois, le plus faible taux moyen de mortalité $(8,93 \pm 2,38 \%)$ a été enregistré dans le bocal témoin négatif en ce qui concerne la variété de riz WAB56-104. Quant au taux de mortalité le plus élevé $(100 \pm 0 \%)$ pour cette variété, il a été enregistré dans les doses $0,75 \mathrm{~g} ; 1 \mathrm{~g}$ et 2,5. Ce taux de mortalité est statistiquement comparable ceux obtenus avec les doses de $0,5 \mathrm{~g} ; 1,4 \mathrm{~g} ; 1,4 \mathrm{~g}$ et $1,85 \mathrm{~g}$ de la poudre de $P$. guineense et le traitement à l'huile de neem.

Tableau I. Effet des doses de P. guineense sur le taux de mortalité de S. oryzae

\begin{tabular}{lll}
\hline & \multicolumn{2}{l}{ Taux moyen de mortalité de S. oryzae $(\%)$} \\
\hline & Oryza sativa, & Oryza sativa, \\
Doses de $P$. guineense & Var. NERICA 1 & Var. WAB56-104 \\
\hline $0,25 \mathrm{~g}$ & $8,35 \pm 1,5 \mathrm{c}$ & $26,83 \pm 1,9 \mathrm{~b}$ \\
$0,50 \mathrm{~g}$ & $41,7 \pm 12,9 \mathrm{~b}$ & $66,92 \pm 8,4 \mathrm{a}$ \\
$0,75 \mathrm{~g}$ & $72,1 \pm 14,2 \mathrm{a}$ & $100 \pm 0 \mathrm{a}$ \\
$1 \mathrm{~g}$ & $99,38 \pm 0,63 \mathrm{a}$ & $100 \pm 0 \mathrm{a}$
\end{tabular}




\begin{tabular}{lll}
$1,4 \mathrm{~g}$ & $98,2 \pm 0,59 \mathrm{a}$ & $99,42 \pm 0,58 \mathrm{a}$ \\
$1,85 \mathrm{~g}$ & $98,75 \pm 0,72 \mathrm{a}$ & $99,39 \pm 0,61 \mathrm{a}$ \\
$2,5 \mathrm{~g}$ & $100 \pm 0 \mathrm{a}$ & $100 \pm 0 \mathrm{a}$ \\
Témoin négatif $(0 \mathrm{~g})$ & $2,99 \pm 0,36 \mathrm{c}$ & $8,93 \pm 2,38 \mathrm{c}$ \\
Huile de neem $(0,5 \mathrm{ml})$ & $83,9 \pm 11 \mathrm{a}$ & $94 \pm 4,34 \mathrm{a}$ \\
Probabilité $(\mathrm{P}>\mathrm{F})$ & $<0,0001$ & $<0,0001$ \\
\hline
\end{tabular}

Les moyennes suivies des mêmes lettres dans chaque colonne ne sont pas significativement différentes selon le test de SNK au seuil de $5 \%$.

\section{Effet de différentes doses de la poudre de $P$. guineense sur l'émergence de $S$. oryzae en fonction des variétés de riz}

L'analyse du tableau 2 montre qu'au $3^{\text {ème }}$ mois de stockage de NERICA 1, toutes les doses de la poudre de $P$. guineense et la dose à l'huile de neem ont significativement réduit le nombre de $S$. oryzae adultes émergés comparé au témoin non traité. Le nombre le plus élevé de charançons de riz adultes émergés $(592,8 \pm 58,4)$ a été obtenu dans le témoin non traité, tandis qu'aucun $S$. oryzae adulte n'a émergé dans les doses $1,85 \mathrm{~g}$ et $2,5 \mathrm{~g}$ de la poudre de $P$. guineense. Toutefois, les nombres de charançons de riz adultes émergés enregistrés dans les doses $0,75 \mathrm{~g} ; 1 \mathrm{~g} ; 1,4 \mathrm{~g} ; 1,85 \mathrm{~g} ; 2,5 \mathrm{~g}$ de la poudre de $P$. guineense ainsi que celui obtenu dans l'huile de neem ne sont pas significativement différents entre eux (Tableau II).

Quant à WAB56-104, au troisième mois après traitement, les nombres d'adultes de $S$. oryzae dans toutes les doses de la poudre de $P$. guineense y compris le témoin à l'huile de neem ont significativement été réduits comparés à la population observée chez le témoin non traité (Tableau II). Il n'y a pas de différence significative entre les populations de $S$. oryzae enregistrées dans les doses $0,5 \mathrm{~g} ; 0,75 \mathrm{~g} ; 1 \mathrm{~g} ; 1,4 \mathrm{~g} ; 1,85 \mathrm{~g}$ et $2,5 \mathrm{~g}$ de la poudre de $P$. guineense ainsi que dans le traitement à l'huile de neem. Au terme du stockage, $634,41 \pm 22,6 \mathrm{~S}$. oryzae adultes émergés ont été dénombrés chez le témoin non traité (Tableau II). Aucun charançon de riz n'a émergé dans la dose de 2,5 g de la poudre de $P$. guineense après trois mois de stockage.

Tableau II. Effet de différentes doses de $P$. guineense sur le nombre moyen de

S. oryzae émergés

\begin{tabular}{lll}
\hline & \multicolumn{2}{c}{ Nombre moyen de S. oryzae émergés } \\
\hline Doses de P. guineense & Oryza sativa, & Oryza sativa, \\
& Var. NERICA 1 & Var. WAB 56-104 \\
\hline $0,25 \mathrm{~g}$ & $420,6 \pm 81,3 \mathrm{~b}$ & $190,86 \pm 24,3 \mathrm{~b}$ \\
$0,50 \mathrm{~g}$ & $93,3 \pm 63,2 \mathrm{c}$ & $22,04 \pm 4,9 \mathrm{c}$ \\
$0,75 \mathrm{~g}$ & $17,13 \pm 7,7 \mathrm{~d}$ & $0,4 \pm 0,5 \mathrm{c}$ \\
$1 \mathrm{~g}$ & $5 \pm 4,83 \mathrm{~d}$ & $0 \pm 0 \mathrm{c}$ \\
$1,4 \mathrm{~g}$ & $1 \pm 0,612 \mathrm{~d}$ & $0,31 \pm 0,38 \mathrm{c}$ \\
$1,85 \mathrm{~g}$ & $0 \pm 0 \mathrm{~d}$ & $0,12 \pm 0,13 \mathrm{c}$ \\
$2,5 \mathrm{~g}$ & $0 \pm 0 \mathrm{~d}$ & $0 \pm 0 \mathrm{c}$ \\
Témoin non traité $(0 \mathrm{~g})$ & $592,8 \pm 58,4 \mathrm{a}$ & $634,41 \pm 22,6 \mathrm{a}$ \\
$0,5 \mathrm{ml}$ d'huile de neem & $10,38 \pm 5,25 \mathrm{~d}$ & $0,79 \pm 1,13 \mathrm{c}$
\end{tabular}


Probabilité $(\mathrm{P})$

$<0,0001$

$<0,0001$

Les moyennes suivies des mêmes lettres dans chaque colonne ne sont pas significativement différentes selon le test de SNK au seuil de $5 \%$.

\section{Effet de différentes doses de la poudre de $\boldsymbol{P}$. guineense sur la teneur en eau des graines}

La teneur en eau des graines de la variété NERICA 1 a été de $9,4 \pm 0,24 \%$ en début de l'expérimentation (Tableau III). Au terme de 3 mois de stockage, la différence entre la teneur en eau des graines a été significative entre tous les traitements. Cette teneur en eau a augmenté dans tous les traitements. Bien plus, les grains issus des pots traités avec la dose $0,5 \mathrm{~g}$ de la poudre de $P$. guineense ont enregistré la teneur la plus élevée en eau $(13,24 \pm 0,21 \%)$, tandis que la plus faible $(11,71 \pm 0,10 \%)$ a été observée dans les pots de $1,4 \mathrm{~g}$ de poudre.

Quant à la variété WAB 56-104, au début de l'expérimentation, la teneur en eau des graines a été de 9,8 $\pm 0,07 \%$ (Tableau III). Après 3 mois de conservation, il a été noté une différence hautement significative en teneur d'eau $(\mathrm{P}<0,0001)$ entre les grains de tous les traitements. L'analyse des données révèle qu'il n'existe aucune différence de teneur en eau entre les traitements à doses de $0,5 \mathrm{~g} ; 0,75 \mathrm{~g} ; 1 \mathrm{~g} ; 1,4 \mathrm{~g} ; 1,85 \mathrm{~g} ; 2,5 \mathrm{~g}$ et le témoin non traité $(\mathrm{P}>0,5)$. La teneur en eau des grains la plus faible $(10,8 \pm 0,21 \%)$ a été obtenue dans le témoin à l'huile de neem, tandis que la plus élevée $(13,4 \pm 0,28$ $\%)$ a été enregistrée dans le témoin non traité et le pot à $2,5 \mathrm{~g}$ de poudre de $P$. guineense (Tableau III).

Tableau III : Effet de différentes doses de la poudre de $P$. guineense sur la teneur en eau des graines

\begin{tabular}{lll}
\hline & Teneur en eau des grains (TE) $(\%)$ \\
\hline & Oryza sativa, & Oryza sativa, \\
Doses de $P$. guineense & Var. NERICA 1 & Var. WAB 56-104 \\
\hline Avant traitements & $9,4 \pm 0,24 \%$ & $9,8 \pm 0,07 \%$ \\
\hline $0,25 \mathrm{~g}$ & $12,46 \pm 0,08 \mathrm{ab}$ & $11,34 \pm 0,31 \mathrm{~b}$ \\
$0,50 \mathrm{~g}$ & $13,23 \pm 0,208 \mathrm{a}$ & $12,65 \pm 0,23 \mathrm{a}$ \\
$0,75 \mathrm{~g}$ & $12,10 \pm 0,454 \mathrm{~b}$ & $12,77 \pm 0,35 \mathrm{a}$ \\
$1 \mathrm{~g}$ & $12,07 \pm 0,253 \mathrm{~b}$ & $12,98 \pm 0,37 \mathrm{a}$ \\
$1,4 \mathrm{~g}$ & $11,71 \pm 0,101 \mathrm{~b}$ & $13,12 \pm 0,10 \mathrm{a}$ \\
$1,85 \mathrm{~g}$ & $11,83 \pm 0,334 \mathrm{~b}$ & $13,24 \pm 0,03 \mathrm{a}$ \\
$2,5 \mathrm{~g}$ & $11,82 \pm 0,0323 \mathrm{~b}$ & $13,4 \pm 0,10 \mathrm{a}$ \\
Témoin non traité $(0 \mathrm{~g})$ & $12,62 \pm 0,276 \mathrm{ab}$ & $13,4 \pm 0,28 \mathrm{a}$ \\
$0,5 \mathrm{ml}$ d'huile de neem & $12,42 \pm 0,12 \mathrm{ab}$ & $10,8 \pm 0,21 \mathrm{~b}$ \\
Probabilité $(\mathrm{P})$ & $<0,008$ & $<0,0001$ \\
\hline
\end{tabular}

Les moyennes suivies des mêmes lettres dans chaque colonne ne sont pas significativement différentes selon le test de SNK au seuil de $5 \%$. 


\section{Effet de différentes doses de la poudre de $P$. guineense sur le taux de germination des graines}

L'effet des doses de la poudre de $P$. guineense sur le taux de germination dépend des variétés. La mesure des taux de germination chez la variété NERICA1 révèle qu'au début de l'expérimentation, cette variété avait un taux de germination de 96,5 $\pm 4,79 \%$ (Tableau IV). Les doses de la poudre de $P$. guineense ont significativement maintenu le taux de germination des grains à un seuil supérieur à celui du témoin non traité. En effet, 3 mois après traitement, le taux de germination le plus élevé $(64 \pm 1,83 \%)$ a été obtenu au niveau de la dose $1 \mathrm{~g}$ de la poudre de $P$. guineense tandis que le témoin non traité a enregistré le taux de germination de 27,5 $\pm 6,18 \%$.

Quant à la variété WAB 56-104 au début de l'expérimentation un taux de germination de $82 \pm 1,15 \%$ a été enregistré dans le lot de semences. Au terme de trois mois de stockage, les taux de germination observés sur tous les différents traitements, sont statistiquement identiques (Tableau IV).

Tableau IV : Effet de différentes doses de la poudre de $P$. guineense sur le taux de germination des graines

\begin{tabular}{lll}
\hline \multicolumn{3}{l}{ Pourcentage $(\%)$ de germination des graines } \\
\hline & Oryza sativa, & Oryza sativa, \\
Doses de $P$. guineense & Var. NERICA 1 & Var. WAB 56-104 \\
\hline Avant traitements & $96,5 \pm 4,79 \%$ & $82 \pm 1,15 \%$ \\
\hline $0,25 \mathrm{~g}$ & $42,5 \pm 5,25 \mathrm{abc}$ & $66,23 \pm 4,99 \mathrm{a}$ \\
$0,50 \mathrm{~g}$ & $34 \pm 6,48 \mathrm{bc}$ & $69,46 \pm 2,16 \mathrm{a}$ \\
$0,75 \mathrm{~g}$ & $54 \pm 7,62 \mathrm{ab}$ & $66,34 \pm 3,86 \mathrm{a}$ \\
$1 \mathrm{~g}$ & $64 \pm 1,83 \mathrm{a}$ & $76,16 \pm 2,74 \mathrm{a}$ \\
$1,4 \mathrm{~g}$ & $59,5 \pm 3,1 \mathrm{a}$ & $82,43 \pm 2,87 \mathrm{a}$ \\
$1,85 \mathrm{~g}$ & $59 \pm 4,51 \mathrm{a}$ & $73,76 \pm 4,9 \mathrm{a}$ \\
$2,5 \mathrm{~g}$ & $55 \pm 8,19 \mathrm{ab}$ & $75,38 \pm 3,5 \mathrm{a}$ \\
$0 \mathrm{~g}$ & $27,5 \pm 6,18 \mathrm{c}$ & $61,68 \pm 3,29 \mathrm{a}$ \\
$0,5 \mathrm{ml}$ d'huile de neem & $47 \pm 3,87 \mathrm{ab}$ & $59,18 \pm 1,4 \mathrm{a}$ \\
Probabilité (P) & 0,000 & 0,238 \\
\hline
\end{tabular}

Les moyennes suivies des mêmes lettres dans chaque colonne ne sont pas significativement différentes selon le test de SNK au seuil de $5 \%$.

\section{Discussion}

Les résultats obtenus de cette étude permettent de dire que la poudre de $P$. guineense serait toxique ou aurait un effet létal sur $S$. oryzae ainsi qu'un effet limitant sur l'apparition de sa progéniture en stock de riz paddy. Ces résultats corroborent ceux de Akunne et al. (2014), Gbaye et al. (2015), et de Akunne et Ononye (2015), qui ont démontré l'activité insecticide et l'efficacité de cette poudre de $P$. guineense, contre $S$. oryzae, ainsi que sur la réduction de sa progéniture en stock de riz. L'effet insecticide de $P$. guineense pourrait être due à l'action des principaux composés tels que la pipérine et le chavicine dont l'effet insecticide a été prouvé par Okonkwo et Okoye (1996). Outre l'effet mécanique que ce poudrage peut avoir (dessèchement, 
obturation des stigmates...). Selon Scott et al. (2004), la pipérine est le principal amide actif chez $P$. guineense. Le mécanisme exact de l'activité de ces produits végétaux à effet insecticide contre les charançons n'est pas très clair, mais ils pourraient impliquer une abrasion physique de la cuticule de l'insecte, entraînant une perte de fluide corporel ou un blocage du spiracle Ogunwolu et al., (1998).

L'effet de la poudre de $P$. guineense dans la réduction de l'apparition de la progéniture de $S$. oryzae pourrait s'expliquer d'une part, par le fait que la poudre du poivre noir exercerait des effets limitants sur la reproduction de S. oryzae (Oparaeke and Bunmi, 2006) ; Upadhyay et Jaiswal, 2007) ; soit en tuant le plus vite possible l'adulte de $S$. oryzae sans leur laisser le temps de se reproduire (Devi and Devi ; 2013), soit en inhibant la fertilité des œufs (Mohd et al. 2014), soit en perturbant la mobilité des adultes en empêchant ainsi leur accès aux grains pour la ponte et la nourriture ou pour la poursuite de leur développement (Taleb-Toudert, 2015).

Par ailleurs, la poudre de $P$. guineense a montré une persistance d'action de 3 mois sur les 2 variétés. Ces résultats sont comparables à ceux obtenus par Sighamony et al. (1984) qui ont prouvé que la persistance d'action de l'extrait de $P$. nigrum pouvait dépasser largement 2 mois dans le contrôle de S. oryzae sur du blé stocké. Selon Abraz (2015), l'hypothèse de forte persistance d'action du poivre noir résulterait d'un mécanisme autre que le contact. Pour cet auteur, cette rémanence pourrait être liée à l'activité des huiles du poivre noir contenue dans les fines particules de sa poudre.

Les taux élevés de germination des graines traitées aux plus fortes doses du poivre noir après 3 mois de stockage s'expliqueraient par le fait que ces doses ont engendré les plus forts taux de mortalité, limité l'apparition de progéniture d'insectes et les dégâts comparativement aux témoins et aux plus faibles doses. Après l'attaque des insectes, la vigueur et le pouvoir germinatif des semences peuvent être altérés (Cruz et al., 1988). C'est ce qui a été observé dans les contrôles avec des taux de germination très faible après 3 mois. A ce temps d'observation, la légère chute du taux de germination des plus fortes doses par rapport aux taux de germination initiaux observés serait liée à l'augmentation de la teneur en eau observée en ce temps, par rapport à la teneur initiale. Ces résultats sont semblables à ceux de Kameswara et al., (2006), qui rapportent que de faibles changements du taux d'humidité ont des effets importants sur la vie en stockage. Cette augmentation de la teneur en eau des grains pourrait être due à la capacité de la poudre de $P$. guineense de capter facilement l'humidité atmosphérique qui sera aussi communiquée aux grains de riz. En effet d'après le site http://icpe.in/icpefoodnpackaging/pdfs/16_spices.pdf, les épices, en particulier celles en poudre, sont de nature hygroscopique et captent l'humidité atmosphérique entraînant l'agglomération de la poudre. Une 
diminution du taux de germination du riz a été observée par Dedi et Allou (2015), ainsi que par Aidani (2015), sur le blé après plusieurs mois de stockage. Pour ces auteurs, également, cette observation serait liée à l'infestation par les insectes qui pour eux est le facteur le plus important qui affecte la germination. Cette hypothèse s'appuie sur les observations faites par Raoelijaona (1996), qui a révélé que la larve de $S$. oryzae, lors de son développement à l'intérieur du grain, dévore aussi bien le germe que l'albumen. Il restera alors un grain vidé présentant un trou (trou de sortie de l'adulte) et contenant les déjections du développement larvaire. Selon Aidani (2015), les dommages causés à l'embryon d'une semence empêchent généralement la germination, car certains ravageurs, comme le charançon du riz s'attaquent de préférence au germe.

\section{Conclusion}

$\mathrm{Au}$ terme de cette étude, il ressort que la persistance d'action de la poudre de $P$. guineense, dans le temps est fonction de la dose appliquée et de la variété de riz utilisée. Les doses 1,4 g et 1,85 g, sont les doses optimales qui ont montré une conservation du taux de germination des grains. Ces doses ont donné une meilleure rémanence de la poudre de $P$. guineense respectivement sur les variétés de riz WAB 56-104 et NERICA 1 pour la lutte contre de $S$. Oryzae et sa progéniture. Enfin ces doses ont permis la maintenance de la faible teneur en eau des grains et du taux de germination à des seuils meilleurs que ceux du témoin négatif. Par ailleurs, l'évaluation des paramètres physiques et agronomiques après traitements, permet de dire que la poudre de $P$. guineense a donc réduit la nuisibilité de $S$. oryzae sur les graines de riz et préservé ainsi relativement leur intégrité germinatif.

\section{References:}

1. Abraz, F. (2015). Étude de l'activité biologique des extraits de quelques plantes à l'égard d'un insecte ravageur des grains stockés, Callosobruchus maculatus (Coleoptèra Bruchidae), mémoire de magister en biologie, Faculté des Sciences Biologiques et Agronomiques, Université de Mouloud Mammeri de IZI Onzoun, Algérie. 69p.

2. AfricaRice (2015). Investing in rice research and innovation for Africa. Annual Report. 36p.

3. Aidini, H. (2015). Effet des attaques de Capucin des grains (Rhizopertha dominica) sur les céréales stockées. "Estimation sur la perte pondérale et le pouvoir germinatif. Cas de blé dur dans la région de Tlemcen ». Mémoire de master en Agronomie, Faculté des Sciences de la nature et de la Vie et des Sciences de la Terre de l'Univers. Université Abou Bekr Belkaid. 104p. 
4. Akunne, C. E. \& Ononye, B. U. (2015). Entomocidal effect of a local spicy and fruit peel Sitophilus oryzae (L.) Coleoptera: Curculionidae Sitophilus oryzae (L.) Coleoptera: Curculionidae. Journal of Entomology and Zoology Studies, 3(3): 208-211.

5. Akunne, C. E., Ononye, B. U., Mogbo, T. C. \& Ngenegbo, U. C. (2014). Evaluation of Zingiber officinale (Schum and Thonn) and Piper guineense (Roscoe) Powders for the Control of Sitophilus oryzae (L.) (Coleoptera: Curculionidae). Journal Sciences Life, 2(3): 342-348.

6. Allagbé, M. C. (2014). Analyse des déterminants de l'adoption des innovations technologiques et de la compétitivité de la riziculture au Bénin. Thèse de doctorat. Faculté des Sciences Agronomiques, Université d'Abomey-Calavi, 194p.

7. Arnason, J.T. \& Philogene, B.J.R. (2004). Efficacy of Piper (Piperaceae) extracts for control of common home and garden insect pests. Journal. Econ. Entomol., 97: 1390-1403.

8. Ashouri, S. \& Shayesteh, N. (2010). Insecticidal activities of two powdered spices, black pepper and red pepper on adults of Rhyzopertha dominica (F.) and Sitophilus granaries (L.). Munis Entomology \& Zoology, 5 (2): 600-607.

9. Atwal, A.S. \& Dhaliwal, G.S. (2002). A text book on Agricultural Pests of South East Asia and their Management. Kalyani Publishers, Ludhiana, 177- 434pp.

10. Benhalima, H., Chaudhry M. Q., Mills, K. A. \& Price N. R. (2004). Phosphine resistance in stored product insects collected from various grain storage facilities in Morocco. Journal. Stored Prod. Res., 40 : 241-249.

11. Cheikh, T. (2008). Bioactivite des extraits de Calotropis procera Ait. et de Senna occidentalis L. sur Caryedon serratus (Ol.), ravageur des stocks et semences d'arachide au Sénégal. Thèse de Doctorat. Université Cheikh Anta Diop, Faculté des Sciences et Technique. Département de Chimie et biochimie des produits naturels, Dakar, Sénégal. 196 p.

12. Cruz, J.F., Troude, F., Griffon, D. \& Hebert, J.P. (1988). Conservation des grains en régions chaudes. 2. éd Paris, France, ministère de la Coopération et du Développement. 545p.

13. Dedi, J. \& Allou, K. (2015). Etude du pouvoir germinatif de quatre variétés de riz que sont GIZA 178, WAB 56-50, LOHININI, DANANE et identification des champignons présents sur les grains en germination. Journal Afrique Science, 11(3) : 161 - 171

14. Devi, K. C. \& Devi, S. S. (2013). Insecticidal and oviposition deterrent properties of some spices against Coleoptera beetle, Sitophilus oryzae. Journal Food Sci Technol. 50 (3): 600-604. 
15. FAOSTAT (2015). FAO Statistical Service, Available at: http://faostat.fao.org/site/291/ default. Aspx United Nations Food and Agriculture Organization, consulté le 30 juillet 2017.

16. Gbaye, O. A., Oyeniyi, E. A. \& Adekanmbi, F. (2015). The Efficacy of Three Plant Powders as an Entomocide against Sitophilus oryzae (Linnaeus) Infesting Rice Grains in Nigeria. International Journal of Research Studies in Zoology (IJRSZ), 1(1): 30-35

17. Gounse, Y. (2004). Analyse socio-économique de la commercialisation du riz local au Centre-Bénin. Thèse d'Ingénieur. Faculté des Sciences Agronomiques, Université d'Abomey-Calavi, Bénin, 137p

18. Guèye, M.T., Seck, D., Wathelet, J-P. \& Lognay, G. (2011). Lutte contre les ravageurs des stocks de céréales et de légumineuses au Sénégal et en Afrique occidentale : synthèse bibliographique. Biotechnol. Agron. Soc. Environ., 15(1): 183-194.

19. Hazoume, J. S. E. (2008). Test d'efficacité de différents traitements non chimiques dans la gestion intégrée des insectes de stocks du riz, mémoire d'Ingénieur agronome, Faculté des Sciences Agronomiques, Université d'Abomey-Calavi, $61 \mathrm{p}$.

20. Helbig, J (1995). The ecology of Prostephanus truancatus in Togo with particular emphasis on interaction with the predator Teretriosoma nigrescens. Deutsche Geselshaft Techniche Zusammenarbeit (GTZ) GmbH, Eschborn, Germany. 111p http://icpe.in/icpefoodnpackaging/pdfs/16_spices.pdf,

21. Juliano, B.O. \& Duff, B. (1989). Setting priorities for rice grain quality research. Paper presented at 12th ASEAN Technical Seminar on Grain Postharvest Technology, Surabaya, Indonesia, 29-31 August

22. Kameswara RN, Hanson J, Ehsan DM., Kakoli G, Nowell D. and Larinde M. (2006). Manuel de manipulation des semences dans les banques de gènes No 8. 166p.

23. MAEP (2013). Direction de la programmation et de la prospective. Rapport de Performance du Secteur Agricole, Cellule de suiviévaluation. Cotonou Bénin. 47p.

24. Mehrpour, O., Karrari P., Zamani, N., Tsatsakis A.M. \& Abdollahi, M. (2014). Occupational exposure to pesticides and consequences on male semen and fertility: A review. Toxicol Lett., 230(2) :146-156.

25. Mohd, A., Kavita, P., Bushra, R, Mohd, A. G., Usman, A. \& Mansoor, A.S., (2014). Bio-efficacy of some leaf extracts on the inhibition of egg hatching and mortality of Meloidogyne incognita, Archives of Phytopathology and Plant Protection, 47(8): 1015-1021. 
26. Ngamo, L.S.T. \& Hance, T. (2007). Diversité des ravageurs des denrées et méthodes alternatives de lutte en milieu tropical. Tropicultura. 25 (4): 215-220.

27. Ogunwolu, E. O., Igoli, J. O. \& Longs, N. N. (1998). Reduction in reproductive fitness of Callosobruchus maculatus $\mathrm{F}$. exposed to Zanthoxylum zanthoxyloides (Lam.) Waterm. Journal of Herbs spices and Medicinal Plants, 6: 19 - 27.

28. Okonkwo, E. U. \& Okoye, W. I. (1996). The efficacy of four seed powders and the essential oils as protectants of cowpea and maize grains against infestation by Callosobruchus maculatus (Fabricius) (Coleoptera: Bruchidae) and Sitophilus zeamais (Coleoptera: Curculionidae) in Nigeria. International Journal of Pest Management, 42(3): $143-146$.

29. Oparaeke, A.M. \& Bunmi, J.O. (2006). Bioactivity of two powdered spices (Piper guineense schum and thonn and Xylopia aethiopica (Dunal) A. Richards) as homemade insecticides against Callosobruchus subinnotatus (Pic.) on stored bambarra groundnut. Agric. Trop. Subtrop., 39 : 132-135.

30. Raoelijaona C. 1996. Les insectes ravageurs du riz, du maïs stockés et des légumineuses sèches. Recueil des exposés lors du symposium à Toliara du 7 au 11 octobre 1996. Antananarivo : Service de la surveillance phytosanitaire du territoire national

31. Scott, I.M., Jensen, H., Nicol, R., Lesage, L., Bradbury, R., SanchezVindas, P. \& Poveda, L. (2004). Efficacy of Piper (Piperaceae) extracts for control of common home and garden insect pests. J Econ Entomol. Aug., 97(4):1390-403.

32. Statista (2017). Principaux pays producteurs de riz au niveau mondial, selon le volume de production (en millions de tonnes métriques). Le Portail de Statistiques. 1p.

33. Sighamony, S.I.A., Chandrakala, T. S. \& Osmani, Z. (1984). Natural products as repellents for Tribolium castaneum Herbst. Int. Pest Contr., 26 : 156-157

34. Taleb-Toudert, K. (2015). Extraction et caractérisation des huiles essentielles de dix plantes aromatiques provenant de la région de Kabylie (Nord Algérien). Evaluation de leurs effets sur le bruche du niébé Callosobruchus maculatus (Coleoptera : Bruchidae). Thèse de doctorat en sciences Biologiques. UM.M.T.O. 206p.

35. Upadhyay, R. K. \& Jaiswal, G., (2007). Evaluation of biological activities of $P$. nigrum oil against $T$. cataneum. Bull. Insectol., 60, 5761. 